\title{
Medical management adherence as an outcome of genetic counseling in a pediatric setting
}

\author{
Sarah Rutherford, MS ${ }^{1,2}$, Xue Zhang, PhD, MSPH², Carrie Atzinger, MS ${ }^{1,2}$, Jennifer Ruschman, $\mathrm{MS}^{3}$ and \\ Melanie F. Myers, PhD, MS ${ }^{1,2}$
}

Purpose: We sought to determine whether the inclusion of a genetic counselor in an initial pediatric genetics visit had an impact on patient adherence to management recommendations, as compared with initial visits in which only genetics physicians were involved.

Methods: This chart review included 198 pediatric patients seen for their initial visit to the general genetics clinic at the Cincinnati Children's Hospital Medical Center in 2008. Ninety-eight patients were seen by one or more genetics physicians (the non-genetic counselor group), and 100 patients were seen by a geneticist and a genetic counselor (the genetic counselor group). Medical management recommendations and evidence of adherence to recommendations were abstracted from the medical record; adherence rates were compared between the genetic counselor and non-genetic counselor groups.
Results: Adherence was significantly associated with the inclusion of a genetic counselor $(P=0.009)$. Although type of management recommendation had a large impact on adherence, involvement of a genetic counselor significantly improved adherence in all three management recommendation categories.

Conclusion: Involvement of a genetic counselor during an initial pediatric genetics visit may be associated with increased patient adherence. Further research is needed to determine whether this association applies in other genetic counseling settings and whether enhanced adherence results in improved medical outcomes.

Genet Med advance online publication 18 July 2013

Key Words: genetic counseling; geneticist; medical management; outcomes; patient adherence

\section{INTRODUCTION}

Outcome studies of genetic counseling can provide evidence of the value of genetic counselors (GCs). Value in health care, defined as health outcomes achieved per dollar spent, benefits patients, payers, and providers by providing the best patient care while maintaining cost efficiency. ${ }^{1}$ Evidence-based genetic counseling is important to maximizing health outcomes for patients and for the maturity of the profession, so that entities inside and outside the profession have a clear understanding of the goals and expected outcomes of genetic counseling practice. Entities outside the profession, such as state licensing boards, insurance companies, and other health-care providers, may use outcome studies to justify the hiring and reimbursement of GCs. ${ }^{2}$

Although GCs can play a key role in educating patients on treatment, management, and/or screening options (e.g., cancer screening for a patient with a hereditary cancer syndrome and diet interventions for a child with a metabolic disease), unlike physicians, GCs do not provide physical interventions such as surgeries or prescribed diets, which have a direct impact on physical effects of disease. Prevention of genetic diseases or birth defects was a medical outcome associated with prenatal genetic counseling in the 1950s and 1960s. However, such a goal is at odds with the views of many in the community of people with disabilities and their allies and is in conflict with the genetic counseling principle of nondirectiveness as it implies that patients should be directed to terminate affected pregnancies. ${ }^{2}$ As GCs become more involved in the provision of care for patients with treatable genetic disorders and common chronic diseases for which lifestyle or other interventions may reduce disease risk, biomedical outcomes such as morbidity and mortality may become increasingly important measures of outcomes in genetic counseling.

Existing research on genetic counseling outcomes has limitations in measuring the full impact of genetic counseling on patient care. For instance, providing education is a well-established goal of genetic counseling. ${ }^{3}$ However, assessing client knowledge implies a judgment about what information should be important to the client (and therefore remembered), which may not always match the client's actual values. ${ }^{4}$ Risk perception, another variable related to education, is also examined in many studies. ${ }^{5-7}$ However, it is well known that risk perception is influenced by factors outside the control of a GC, such as the client's personality, and can even change with time as the client's life situation changes. ${ }^{4}$ Psychosocial support is another goal of genetic counseling. ${ }^{3}$ Studies have evaluated client satisfaction with counseling and "psychological adjustment" by measuring well-being and perceived personal control. ${ }^{8-11}$ But

${ }^{1}$ Department of Pediatrics, College of Medicine, University of Cincinnati, Cincinnati, Ohio, USA; ${ }^{2}$ Division of Human Genetics, Cincinnati Children's Hospital Medical Center, Cincinnati, Ohio, USA; ${ }^{3}$ Department of Planning and Business Development, Cincinnati Children's Hospital Medical Center, Cincinnati, Ohio, USA. Correspondence:

Melanie F. Myers (Melanie.Myers@cchmc.org) 
satisfaction and psychological outcomes are not sufficient to quantify the health effects of genetic counseling. Therefore, the usefulness of these outcomes in evaluating the effectiveness of the GC may be limited, and new outcome measures are needed. ${ }^{2}$

Patient adherence is a crucial link between the process of providing health-care and health outcomes. ${ }^{1}$ Medical management adherence is widely studied in medicine. Factors affecting adherence to medication are multifactorial and include patient-related factors (e.g., age and cognitive impairment), condition-related factors (e.g., severity and type of symptoms), socioeconomic factors (e.g., high treatment costs and low literacy), therapy-related factors (e.g., complexity of the regimen), and health-system factors (e.g., access to healthcare). ${ }^{12}$ Interventions by health-care providers that have been shown to increase patient adherence include taking extended time to clarify instructions, tailoring the regimen to the patient's lifestyle, and providing educational materials to allow the patient to self-educate and improve their self-efficacy. ${ }^{13,14}$

The provider-patient relationship also influences adherence. A relationship based on effective communication and collaboration allows patients to become involved in planning their own care. Involved patients demonstrate better adherence, express greater satisfaction, and have better control of their chronic conditions. ${ }^{14,15}$ The reciprocal engagement model of genetic counseling proposes that "the genetic counselor and patient make decisions in a collaborative fashion-there is a give and take between counselor and patient." 16 Because patients involved in collaborating on their medical decisions demonstrate higher satisfaction and better adherence, facilitation of collaborative decision making by GCs may be associated with increased patient adherence.

Patients are also more likely to adhere to their treatment plan when their provider demonstrates empathy and builds interpersonal trust. ${ }^{15}$ As part of their practice, GCs are expected to provide psychosocial counseling, which is influenced by the principles of Carl Rogers' client-centered counseling. The use of empathy is a main principle of client-centered counseling. Therefore, measurement of medical management adherence may correlate with the provision of empathy and psychosocial counseling in a genetic counseling session. Alternatively, adherence could be associated with provider practice patterns, such as frequency of patient follow-up. However, client adherence to medical management recommendations has not been previously studied as an outcome in genetic counseling.

The purpose of this study was to determine whether the inclusion of a GC in a pediatric patient's initial genetics visit had an impact on patient adherence to medical management recommendations. Specifically, we examined the probabilities that patients completed medical management recommendations when a GC was involved in the patient's initial visit versus when only genetics physicians were involved. As a secondary outcome, we also compared the number of concepts recorded in the psychosocial section of the medical intake form following an initial visit to a general genetics clinic.

\section{MATERIALS AND METHODS}

This study was approved by the University of Cincinnati institutional review board and the Cincinnati Children's Hospital Medical Center institutional review board. Medical records of 198 pediatric patients (12 years of age or younger) seen for their first visit to the general genetics clinic at the Cincinnati Children's Hospital Medical Center (CCHMC) between 1 January 2008 and 31 December 2008 were abstracted. Exclusion criteria included patients seen by a genetics specialist at a nonCCHMC location before their initial visit to the CCHMC, patients seen in a specialty genetics clinic (e.g., neurofibromatosis clinic) or referred to a general genetics clinic from an inpatient genetics consult at CCHMC, patients seen by a nurse or nutritionist as part of their initial visit, and patients who were not seen by an attending clinical geneticist.

A database of patients seen by the Division of Human Genetics at the CCHMC was used to identify patients who fulfilled the inclusion criteria. Of the 538 patients who met the inclusion criteria, 317 had a GC involved in their initial visit and 219 did not. The inclusion of a GC in genetics appointments at the CCHMC during the time frame of this study was primarily based on nonpatient factors such as clinic schedule and GC availability. Due to growth in specialty clinics and patient volumes during this time period, genetic counseling staff were not scheduled to attend all general genetics clinics. New-visit appointments were not scheduled differently based on whether a GC was or was not available to see the patient; nor were indications for referral a reason to schedule patients differently. New-visit appointments were scheduled for $60 \mathrm{~min}$; however, actual length of clinic visits was not systematically recorded.

Descriptive statistics were calculated to determine the age and sex distributions of patients fulfilling the inclusion criteria. Age was categorized on the basis of stages of child development as follows: infant, $0-12$ months; toddler, 1 year and 1 day to 5 years; and school age, 5 years and 1 day to 12 years.

One hundred patients were randomly selected from the population of patients who had seen a GC and a clinical geneticist-physician team; this group is designated as the GC group. Patients who had seen a clinical geneticist (with or without the addition of a medical genetics resident), but not a GC, were selected and matched to the GC group on the basis of age category and sex; this group is designated the non-GC group. A unique identification number was assigned to each patient, and an Excel document was created to link the medical record number to the unique identification number created for each patient. If review of the medical record indicated that a patient did not fulfill the inclusion criteria, the patient was excluded, and another patient was selected. One round of reselection was performed; if the re-selected patient did not fulfill the inclusion criteria, the patient was excluded.

A paper data-abstraction form was created to collect data from the medical record including demographic information, medical management adherence, and psychosocial concepts addressed during the initial visit. Demographic information included patient age, sex, insurance type, and reason for referral. After 
review by the research team, reason for referral was collapsed into five categories: congenital anomalies, nonspecific symptoms (including developmental delay/cognitive impairment and nonspecific dysmorphic features), prenatal exposures, known diagnosis/family history of a genetic condition, and to rule out a specific genetic condition. The medical record included paper medical charts and data from two electronic medical record systems that were in use at the CCHMC in 2008: Chartmaxx and Epic. All information in the data-abstraction forms were entered into an Access database developed for this study.

\section{Medical management adherence}

The medical management recommendations recorded in each chart, found in the "Plan" section of the dictation from the patient visit, were abstracted. Any statement listed under "Plan" that did not describe a task that the patient was intended to complete was not counted as a recommendation. In places where we refer to "patient adherence," it was assumed that medical management recommendations were made to the parent or adult accompanying the child to the visit and that adherence was facilitated by this parent or adult because this was a pediatric population.

Medical management recommendations included follow-up with genetics, referral to a specialist (cardiology, craniofacial, developmental, and behavioral pediatrics; endocrinology; otolaryngology; feeding; gastroenterology; neurology; ophthalmology; orthopedics; and other), genetic testing (karyotype, microarray, sequencing, and other), imaging (magnetic resonance imaging, computed tomography scan, ultrasound, X-ray, and other), other testing or screening therapies (physical, occupational, or speech), school recommendations (e.g., change of schools), lifestyle recommendations (e.g., start exercise plan), and requests for medical records from other institutions. After review by the research team, these categories of recommendations were further collapsed into three main themes, including "follow-up with genetics," which included any recommendation for the patient to return to genetics; "referral to specialist," which included any recommendation for the patient to see a health-care professional outside of genetics; and "testing," which included any recommended follow-up testing such as genetic testing, imaging, and other blood, urine, or sweat tests. Recommendations that did not fit these categories were omitted (GC: $n=17$; non-GC: $n=25$ ) because they were activities to be completed outside the medical setting, and thus a medical record review was not a good way to find evidence of adherence. The omitted types included school recommendations, lifestyle changes, and requests for medical records from other institutions.

For each recommendation made, an attempt was carried out to locate evidence in the medical record that the recommendation was completed. Evidence included test results, documentation of attendance at recommended appointments with genetics or other specialists at the CCHMC, and documentation of follow-up with appointments or care outside of the CCHMC (e.g., written documentation of the parents' verbal report of attending appointments). Referrals were considered complete if the patient showed up for an appointment with the specialist to which he or she was referred. Recommendations were considered complete only if they were completed within 18 months of the initial visit. If no evidence of follow-up was found, it was assumed that the recommendation was not completed.

\section{Psychosocial concepts}

The "psychosocial" section of the intake form was examined, and the number and type of psychosocial concepts recorded in the medical record was entered into the data-abstraction form. The 11 types of psychosocial concepts recorded based on consultation with the research team included with whom the patient lived; family support systems; family structure and relationships; school issues; insurance and finances; religion/culture; parents' work; coping skills; parent/patient feelings; ability to travel to medical appointments; and patient interests and hobbies.

\section{Statistical analysis}

Statistical analyses were performed using Statistical Analysis Software (SAS), version 9.3 (SAS Institute, Cary, NC). Because a single patient could have more than one recommendation, to account for the within-subject correlation, we used a generalized estimating equation model to assess the impact of involvement of GCs on the completion of recommendations.

The number of psychosocial concepts was compared between the GC and non-GC groups using linear regression.

The effects of patient age, sex, insurance type, and reason for referral group were tested for both the outcomes; the effect of recommendation type was also tested for the adherence outcome, as was the presence of a medical resident as compared with a GC. Variables that showed a significant impact on outcomes were included in the final models. Associations were considered significant when $P \leq 0.05$.

\section{Interrater reliability}

To increase reliability, a second coder re-abstracted 40 of the 198 patient records. Cohen's $\kappa$ was then used to assess the interrater agreement on the three recommendation types and the 11 psychosocial concepts.

\section{RESULTS}

The final sample of patients for the GC group $(n=100)$ consisted of 14 female infants, 14 male infants, 18 female toddlers, 22 male toddlers, 14 female school-aged children, and 18 male school-aged children.

The final sample of patients in the non-GC group $(n=98)$ consisted of 13 female infants, 13 male infants, 18 female toddlers, 22 male toddlers, 14 female school-aged children, and 18 male school-aged children. Two patients in the non-GC group were excluded after one round of reselection because they had seen a GC. The distribution is consistent with the age and sex distribution of the population of patients fulfilling the inclusion criteria.

The clinicians involved in the initial visits of the sample population included six clinical geneticists, five medical genetics 
residents, and nine GCs. Three of the six clinical geneticists were female, and two of five residents were female. All of the GCs were female.

Among the GC group, 96 patients had seen a clinical geneticist and a GC and 4 had seen a clinical geneticist, a medical genetics resident, and a GC. Among the non-GC group, 76 patients had seen a clinical geneticist and medical genetics resident during their visit, and 22 had seen only a clinical geneticist. No significant differences were detected in demographics between the GC and non-GC groups (Table 1).

\section{Medical management adherence}

Overall, a similar number of medical management recommendations were made in the GC group $(n=228)$ and the non-GC group $(n=208)$ (Table 2). The number of recommendations in each category (follow-up with genetics, referral to a specialist, and testing) was also similar between the two groups. Percent completion of recommendations appears higher in the GC group than the non-GC group (Table 2). Interrater reliability (determined by Cohen's $\kappa$ ) for categorizing the recommendation types was good $(\kappa=0.78)$.

The results from the generalized estimating equation model showed that adherence was significantly associated with the inclusion of a GC $(P=0.009)$; patients in the GC group were more likely to complete their recommendations than patients in the non-GC group (odds ratio $=2.1 ; 95 \%$ confidence

\section{Table 1 Demographics}

\begin{tabular}{|c|c|c|c|}
\hline & $\begin{array}{l}\text { GC group } \\
(n=100)\end{array}$ & $\begin{array}{c}\text { Non-GC group } \\
(n=98)\end{array}$ & $P$ value \\
\hline Age (years) (median (IQR)) & $2.54(0.92-6)$ & $2.21(0.90-6.13)$ & 0.88 \\
\hline $\operatorname{Sex}(n(\%))$ & & & 0.78 \\
\hline Female & $45(45)$ & $46(47)$ & \\
\hline Male & $55(55)$ & $52(53)$ & \\
\hline Insurance $(n(\%))$ & & & 0.18 \\
\hline Public & $24(24)$ & $36(37)$ & \\
\hline Private & $64(64)$ & $51(52)$ & \\
\hline None & $4(4)$ & $6(6)$ & \\
\hline Unknown & $8(8)$ & $5(5)$ & \\
\hline Reason for referral $(n(\%))$ & & & 0.49 \\
\hline Congenital anomalies $^{b}$ & $21(21)$ & $12(12)$ & \\
\hline Nonspecific symptoms ${ }^{c}$ & $27(27)$ & $27(28)$ & \\
\hline Prenatal exposures ${ }^{d}$ & $2(2)$ & $3(3)$ & \\
\hline $\begin{array}{l}\text { Known diagnosis/ } \\
\text { family historye }\end{array}$ & $20(20)$ & $26(27)$ & \\
\hline Rule out disorder ${ }^{f}$ & $30(30)$ & $29(30)$ & \\
\hline
\end{tabular}

GC, genetic counselor; IQR, interquartile range.

${ }^{a}$ Age was compared using Wilcoxon rank sum test; sex, insurance, and reason for referral were compared using $\chi^{2}$ tests. ${ }^{\text {b}}$ Congenital anomalies: includes single or multiple congenital anomalies, e.g., "craniosynostosis," "developmental delay and Poland sequence," "duplicated right thumb," and "multiple congenital anomalies." "Nonspecific symptoms: includes developmental delay/cognitive impairment,

behavior concerns, and nonspecific dysmorphic features. 'Exposures: suspected or known prenatal exposures to drugs and/or alcohol. eKnown diagnosis/family history: includes patients referred for a known diagnosis or a family history of a known genetic condition. 'Rule out: includes referrals that specifically include a syndrome to rule out ("r/o neurofibromatosis") or include symptoms that are strongly suggestive of a specific genetic disorder ("café au lait spots and axillary freckles"). interval $=1.2-3.6)($ Figure 1$)$. Interrater reliability for adherence to recommendations was excellent $(\kappa=0.933)$.

To control for the presence of a second medical provider during a patient visit, we examined whether the presence of a resident with a clinical geneticist $(n=76)$ as compared with a GC with a clinical geneticist $(n=96)$ made a difference in adherence. The number of recommendations completed was significantly higher when a GC was present $(71 \%)$ versus when a resident was present $\left(60 \% ; \chi^{2}=6.86 ; 1\right.$ degree of freedom; $P=0.009$ ). Results were similar using a generalized estimating equation model that controlled for within-subject correlations in adherence and the different types of recommendations $(P=0.006)$.

Adherence was also significantly associated with recommendation type $(P<0.0001)$ with patients least likely to complete a recommendation to follow-up in genetics clinic, and most likely to complete an ordered test (Figure 1).

No association was detected between adherence and patient age, sex, insurance type, or reason for referral group.

\section{Psychosocial concepts}

Cohen's $\kappa$ for agreement on the 11 types of psychosocial concepts was good $(\kappa=0.64)$. Almost all disagreement consisted of one coder including a concept that the other did not code.

Table 2 Total number of recommendations and percent completed, by group and recommendation type

\begin{tabular}{lcccc} 
& \multicolumn{2}{c}{ GC group } & \multicolumn{2}{c}{ Non-GC group } \\
\cline { 2 - 5 } & $\begin{array}{c}\text { Number of } \\
\text { recommen- } \\
\text { dations }\end{array}$ & $\begin{array}{c}\text { Percent } \\
\text { completed } \\
(\boldsymbol{n})\end{array}$ & $\begin{array}{c}\text { Number of } \\
\text { recommen- } \\
\text { dations }\end{array}$ & $\begin{array}{c}\text { Percent } \\
\text { completed } \\
(\boldsymbol{n})\end{array}$ \\
\hline $\begin{array}{l}\text { Follow-up } \\
\text { with genetics }\end{array}$ & 65 & $57 \%(37)$ & 69 & $38 \%(26)$ \\
$\begin{array}{l}\text { Referral to } \\
\text { specialist }\end{array}$ & 38 & $76 \%(29)$ & 37 & $57 \%(21)$ \\
Testing & 125 & $92 \%(115)$ & 102 & $87 \%(89)$ \\
All types & 228 & $79 \%(181)$ & 208 & $65 \%(136)$ \\
\hline
\end{tabular}

$\mathrm{GC}$, genetic counselor.

aRecommendations for follow-up testing, including genetic testing, imaging, and other blood, urine, or sweat tests.

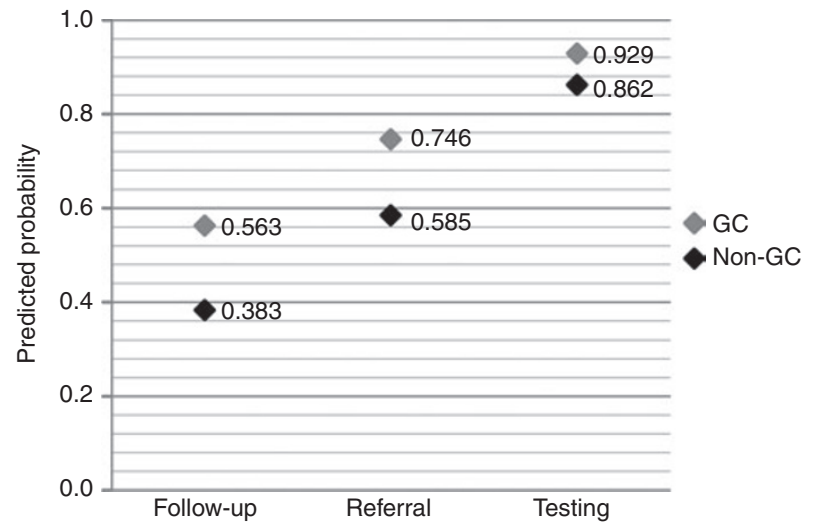

Figure 1 Predicted probability of adherence by recommendation type. 
The number and types of psychosocial concepts recorded in the medical records in the GC and non-GC groups are described in Table 3. Five patient records in the GC group and five patient records in the non-GC group did not include any of the 11 types of psychosocial concepts listed in Table 3.

The mean number of psychosocial concepts recorded per medical record was 2.3 in the GC group (SD: 1.1) and 2.1 in the non-GC group (SD: 0.9). Linear regression results showed a marginal significant difference in the number of psychosocial concepts documented per medical record between the GC group and the non-GC group $(P=0.048)$. After controlling for the demographic variables described in Table 1, more psychosocial concepts were recorded per medical record in the GC group than the non-GC group. Reason for referral category (congenital anomalies; nonspecific symptoms; prenatal exposures; known diagnosis/family history; to rule out disorder) also showed a significant impact on the number of concepts $(P=0.001)$. A significant association was found between patient sex and number of psychosocial concepts recorded $(P=0.001)$, with males having more psychosocial concepts recorded in the medical record than females.
However, no significant difference was detected in concept types between males and females ( $P=0.68 ; \chi^{2}$ test), as illustrated in Figure 2.

\section{DISCUSSION}

\section{Medical management adherence}

Families of general pediatric genetics patients in this study were significantly more likely to adhere to medical management recommendations when they were seen by a geneticist and GC team, as compared with patients seen only by genetics physicians. Although the type of management recommendation had a large impact on adherence, involvement of a GC improved adherence in all three management recommendation categories.

Although we can only speculate as to the reasons for higher adherence among the GC group, the quality of provider-family communication and collaborative decision making have been shown to facilitate treatment adherence, health outcomes, and satisfaction with care in managing pediatric and adult chronic illnesses. ${ }^{14,15,17}$ Patients are more likely to adhere to their treatment plan when their provider demonstrates empathy and

Table 3 Psychosocial concepts by group

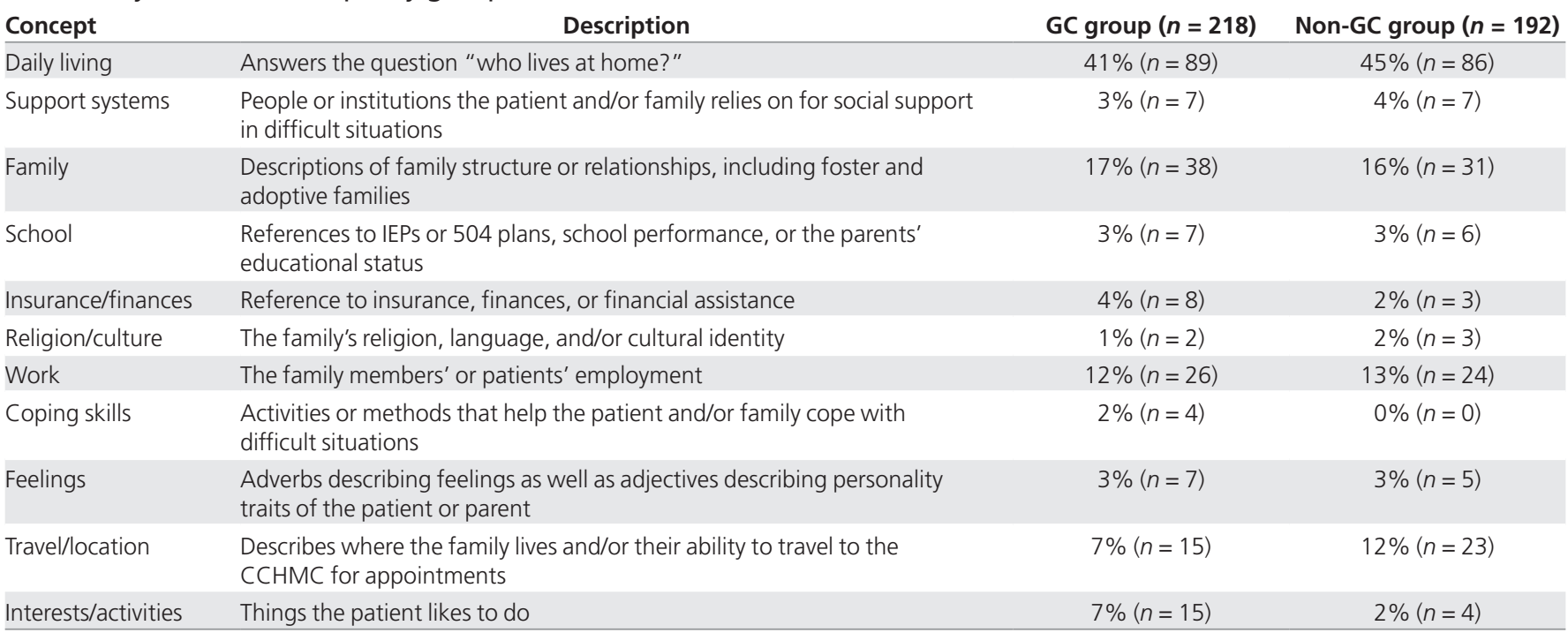

CCHMC, Cincinnati Children's Hospital Medical Center; GC, genetic counselor; IEP, Individual Education Plan.

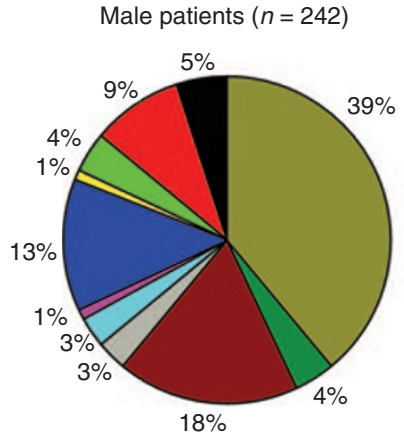

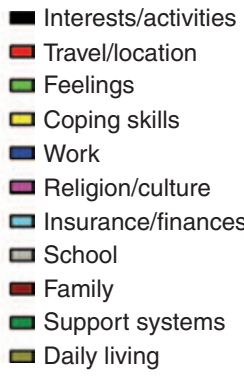

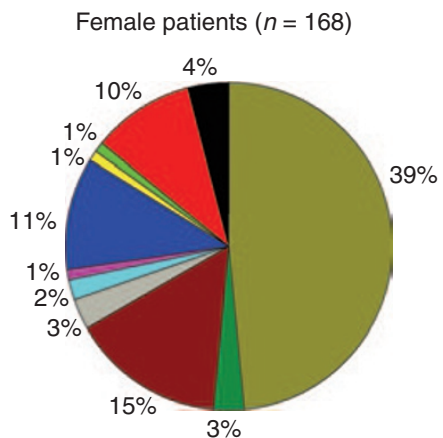

Figure 2 Psychosocial concepts by patient sex. 
builds interpersonal trust. ${ }^{15}$ Relationship-building elements of the genetic counseling process that have been associated with more positive outcomes include higher levels of counselor facilitation of understanding and empathic responses and lower levels of verbal dominance. ${ }^{18,19}$

The specialty expertise of GCs in the area of decision making could have also played a role in parents' adherence to recommendations. In a collaborative decision-making process supported by a nondirective GC, a parent may be more satisfied with the choices they make, and their active involvement may even make them feel more motivated to follow through on medical management recommendations. ${ }^{15,20}$

Patient adherence is a crucial link between care process and health outcomes. ${ }^{1}$ Although the effect of recommendation adherence on medical outcomes has not been previously studied in the field of general pediatric genetics, the effect of adherence to medications and treatments in other fields has been shown to reduce the effects of disease. The use of medical outcomes studied and accepted in other areas of health care may help demonstrate the value of genetic counseling to payers and employers.

\section{Psychosocial concepts}

There was a marginally significant difference in the number of psychosocial concepts recorded per medical record between the GC and non-GC groups. Moreover, reasons for these differences are speculative, but it is possible that GCs discussed more psychosocial concepts with parents than the genetic physicians. Alternatively, the GCs may have been more likely to record the psychosocial concepts they discussed. Previous studies indicate that providers may sometimes omit psychosocial information from the medical record even if they feel it is important, possibly due to concerns of patient privacy. ${ }^{21} \mathrm{~A}$ study of videotaped appointments in internal medicine found that in 12 of 20 consultations, the patients reported psychosocial information that was not documented in the chart. ${ }^{22}$

Our study reports the number of psychosocial concepts documented, but we could not assess the amount of time spent discussing each concept or the depth of the discussion. The provider's use of a counseling skill like empathy would not be recorded in the medical record; however, patient outcomes may be more influenced by the process of psychosocial counseling and the techniques employed by their provider rather than the number of topics discussed. Therefore, differences in the number of concepts recorded may not fully represent whether there is a difference in the quality of the provider-patient relationship between the two groups. Evaluations of psychosocial counseling outcomes may be best performed with a different research model, such as audio- or videotaped sessions, which would be better able to capture all concepts discussed and psychosocial skills employed by the provider.

A significant difference in the number of psychosocial concepts recorded in the medical record was found between male and female patients, with more concepts recorded in the charts of male patients. There was no significant interaction between reason for referral category and sex. However, the difference by sex in documented psychosocial concepts could have been caused by differences in the reason for referral between males and females that were not captured by our five collapsed reasons for referral. Data on whether an X-linked condition was suspected in the patient was not collected, and therefore it was not possible to analyze whether the increased number of psychosocial concepts recorded in males' charts was associated with suspicion of X-linked conditions. Of note, because the mean age of the patients in both the groups was 2 years or younger, any psychosocial counseling provided was almost certainly performed with the parent or guardian who accompanied the child to the visit rather than the patient themselves. Sex of the parent or guardian accompanying the patient was not recorded.

\section{Limitations}

This study analyzed adherence only following an initial visit and did not measure adherence at follow-up visits. Because this study was conducted at a single large pediatric center, whether the findings are generalizable to other genetics centers or genetics settings that include adults is unknown. Calculation of adherence in this study was limited by the ability to find documentation in the CCHMC medical record. However, the recommendation to follow-up with genetics was likely the easiest to find documentation of adherence.

Each patient's reason for referral to genetics was abstracted, and the data were collapsed into five categories. There may have been factors related to reason for referral that were not captured in data abstraction or collapsed category creation. For instance, the family's level of anxiety or perceived severity of the child's condition was not determined, so it was not possible to analyze whether family anxiety influenced adherence or psychosocial concepts recorded. Insurance type was collected as an indicator of socioeconomic status, but the number of patients with no insurance at all was low enough that there may not have been power to detect differences between the no-insurance group and the public and private insurance groups.

Although patient medical records were randomly selected and matched on age and sex, there may be other confounding factors for which we did not control that were associated with adherence. For example, we do not have data on the number of follow-up phone calls to families and by whom they were made. We were also unable to examine whether adherence differed by provider gender because all the GCs were female. Finally, we do not have data on previsit and postvisit provider contact with families. However, it is rare that GCs would have contact with a new-patient previsit. Although it is plausible that individual providers differ in how they track and follow-up on recommendations made to patients, such differences would only be meaningful if they were consistent across provider type. Future studies that randomize participants to the type of health-care provider may be helpful in controlling for such unknown confounders.

This study was not able to identify the reason for increased patient adherence in the GC group, nor was it able to analyze the parents' internal influences or motivations for 
completing recommendations for their child. In addition, although the clinical geneticists, residents, and GCs all likely integrated some elements of genetic counseling into new patient visits, we are not able to retrospectively discern what processes each provider integrated and how they differed by provider type.

\section{Future studies}

This is the first study of adherence by parents of patients referred to genetics as an outcome of genetic counseling and could be used as a model for additional outcome studies in genetic counseling. Additional studies are needed that assess reasons for differences in adherence when a GC is and is not involved in initial pediatric visits. Further research is needed to determine whether the association of GCs with increased parental adherence applies in other genetic counseling settings and at other centers, and if enhanced adherence results in improved medical outcomes and/or lower health-care costs.

We did not have sufficient numbers to assess outcomes when nurses and dieticians were involved in new-patient visits. However, it is possible that nurses and dieticians play similar roles as GCs during patient encounters and may make for an additional comparison group in future studies.

The results from this study also generate further questions as to what processes of genetic counseling influence adherence and how. Genetic counseling process studies could identify provider practices and patient characteristics that are associated with patient or parental adherence. Such studies might involve audio- or video-taping appointments and could also be examined to assess differences in psychosocial counseling. As previous studies have suggested that the patient-provider relationship and collaborative decision making are correlated with adherence; future studies should investigate these associations in a genetic counseling setting.

\section{ACKNOWLDEGMENTS}

The research was conducted at Cincinnati Children's Hospital Medical Center. The authors thank Kimberly Lewis for her assistance in re-abstracting medical records. S.R. received a University of Cincinnati Graduate School Governance Association Student Research Award of US $\$ 400$ for completion of this study.

\section{DISCLOSURE}

The authors declare no conflict of interest.

\section{REFERENCES}

1. Porter ME. What is value in health care? N Eng/ J Med 2010;363:2477-2481.

2. Biesecker BB. Goals of genetic counseling. Clin Genet 2001;60:323-330.

3. Bernhardt BA, Biesecker BB, Mastromarino CL. Goals, benefits, and outcomes of genetic counseling: client and genetic counselor assessment. Am J Med Genet 2000;94:189-197.

4. Clarke A, Parsons E, Williams A. Outcomes and process in genetic counselling. Clin Genet 1996;50:462-469.

5. Lobb EA, Butow PN, Barratt A, et al. Communication and information-giving in high-risk breast cancer consultations: influence on patient outcomes. $\mathrm{Br} J$ Cancer 2004;90:321-327.

6. Meiser B, Butow PN, Barratt AL, et al.; Psychological Impact Collaborative Group. Long-term outcomes of genetic counseling in women at increased risk of developing hereditary breast cancer. Patient Educ Couns 2001;44: 215-225.

7. Rantala J, Platten U, Lindgren $G$, et al. Risk perception after genetic counseling in patients with increased risk of cancer. Hered Cancer Clin Pract 2009;7:15.

8. Davey A, Rostant K, Harrop K, Goldblatt J, O'Leary P. Evaluating genetic counseling: client expectations, psychological adjustment and satisfaction with service. J Genet Couns 2005;14:197-206.

9. Berkenstadt M, Shiloh S, Barkai G, Katznelson MB, Goldman B. Perceived personal control (PPC): a new concept in measuring outcome of genetic counseling. Am J Med Genet 1999;82:53-59.

10. Aalfs CM, Oort FJ, de Haes JC, Leschot NJ, Smets EM. A comparison of counselee and counselor satisfaction in reproductive genetic counseling. Clin Genet 2007;72:74-82.

11. Smets EM, Pieterse AH, Aalfs CM, Ausems MG, van Dulmen AM. The perceived personal control (PPC) questionnaire as an outcome of genetic counseling: reliability and validity of the instrument. Am J Med Genet A 2006;140:843-850.

12. Ho PM, Bryson CL, Rumsfeld JS. Medication adherence: its importance in cardiovascular outcomes. Circulation 2009;119:3028-3035.

13. Chesney MA. Factors affecting adherence to antiretroviral therapy. Clin Infect Dis 2000;30(suppl 2):S171-S176.

14. Martin LR, Williams SL, Haskard KB, Dimatteo MR. The challenge of patient adherence. Ther Clin Risk Manag 2005;1:189-199.

15. Feldman M, Christensen J. Behavioral Medicine: A Guide for Clinical Practice, 3rd edn. McGraw-Hill Medical: New York, 2008.

16. Veach PM, Bartels DM, Leroy BS. Coming full circle: a reciprocal-engagement model of genetic counseling practice. J Genet Couns 2007;16:713-728.

17. Drotar D, Crawford $\mathrm{P}$, Bonner M. Collaborative decision-making and promoting treatment adherence in pediatric chronic illness. Patient Intell 2010;2:1-7.

18. Meiser B, Irle J, Lobb E, Barlow-Stewart K. Assessment of the content and process of genetic counseling: a critical review of empirical studies. J Genet Couns 2008;17:434-451.

19. Roter D, Ellington L, Erby LH, Larson S, Dudley W. The Genetic Counseling Video Project (GCVP): models of practice. Am J Med Genet C Semin Med Genet 2006;142C:209-220.

20. Mendel R, Traut-Mattausch E, Frey D, et al. Do physicians' recommendations pull patients away from their preferred treatment options? Health Expect 2012;15:23-31.

21. Ståhl $Y$, Granlund M, Gäre-Andersson B, Enskär K. Views on health information and perceptions of standardized electronic records among staff in Child and School Health Services. J Nurs Manag 2011;19:201-208.

22. Langewitz WA, Loeb $Y$, Nübling $M$, Hunziker $S$. From patient talk to physician notes-comparing the content of medical interviews with medical records in a sample of outpatients in internal medicine. Patient Educ Couns 2009;76:336-340. 